\title{
REVISÃO DA LITERATURA PARA CONSTRUÇÃO DE UM MODELO DE PADRONIZAÇÃO E INTEGRAÇÃO DE DADOS DE PESQUISAS SOBRE MPE'S DE MINAS GERAIS EM UM REPOSITÓRIO BASEADO EM LINKED DATA
}

Luander Falcão $^{1}$

\section{${ }^{1}$ Sebrae-MG}




\section{Revisão da Literatura para Construção de um Modelo de Padronização e Integração de dados de pesquisas sobre MPE's de Minas Gerais em um repositório baseado em Linked Data}

Resumo: As Pequenas Empresas são a maioria das empresas de um país, empregam no máximo 100 funcionários e normalmente não utilizam os dados internos para subsidiar as decisões. Isso as tornam mais vulneráveis às oscilações do mercado, como quando há impactos econômicos, tecnológicos, regulatórios e socioculturais. Devido a importância das MPE's na economia e no ambiente social, instituições foram criadas para dar apoio às MPE's. Essas instituições realizam várias pesquisas com as MPE's ao longo do ano, para entender esse público. Porém esses dados não estão integrados, logo, não podem ser reutilizados. O uso de ontologia como modelo conceitual para integração de dados em um ambiente Linked Data resolveria isso, além de poder ser relacionado a outros ambientes de compartilhamento de dados. A Revisão da Literatura aponta que há poucas pesquisas que relacionam MPE, Ontologia, Integração de Dados e Linked Data.

Palavras-chave: Pequenas Empresas. Ontologia. Integração de Dados. Linked Data.

\section{$1 \quad$ Introdução}

Em uma economia globalizada e de forte competição, em que os consumidores possuem maior quantidade de informação sobre produtos e serviços, as empresas não podem repetir práticas e estratégias responsáveis por seu sucesso no passado (DAVENPORT; PRUSAK, 2003). As empresas chegaram a este patamar a partir da influência do macro ambiente sobre elas, o que as tornam mais complexas, competitivas, globais e velozes em se adaptarem (SILVA; NAVES, 2004).

Nesse cenário destacamos as empresas de micro e pequeno porte, também denominadas MPE's. Segundo o Sebrae (2019), Nos Estados Unidos são 27,9 milhões de pequenas e médias, ou seja, 99,7\% das empresas americanas. Geram 50\% dos empregos e 50\% do PIB. No Brasil, segundo o Sebrae (2018), são 15,4 milhões de pequenos negócios, que representam $98,5 \%$ do total de empresas, $54 \%$ dos postos de trabalho e $27 \%$ do PIB do país. Os números apontam para a relevância desse tipo de empresa na economia e sociedade de um país.

Tanto nos Estados Unidos como no Brasil há órgãos especializados que visam auxiliar, aconselhar, assistir e proteger os interesses dos empreendimentos de pequeno porte. No Brasil esse órgão é denominado SEBRAE - Serviço Brasileiro de Apoio às Micro e Pequenas Empresas. O Sebrae "é uma entidade privada que promove a competitividade e o desenvolvimento sustentável dos empreendimentos de micro pequenas empresas- aqueles com faturamento bruto anual de até R \$ 4,8 milhões" (SEBRAE, 2019).

O Sebrae atua em todo o território nacional, e conta com pontos de atendimento nas 27 Unidades da Federação. Em cada estado são oferecidos cursos, seminários, consultorias e assistência técnica para pequenos negócios de todos os setores. As unidades que compõe o Sistemas Sebrae realizam no decorrer do ano várias pesquisas, com o objetivo de entender e compreender o seu público alvo, que é disperso e heterogêneo, mesmo dentro de um estado. 
Isso cria uma massa de dados e informações que ficam inertes, após o fim da pesquisa, e não são reutilizadas para responder outras perguntas.

Nesse sentido, para Mcgee e Prusak (1994, p. 23), "a informação cada vez mais será a base da competição". Segundo Choo (2003), a informação pode ser processada e usada com o objetivo de criação de significado, construção do conhecimento e tomada de decisão. Isto gera fluxos de informação e conhecimento, que podem ser internos ou externos, em um contexto de conhecimento interativo, propiciado pelo computador, telecomunicação e convergência tecnológica.

Em um contexto de alta competição e rivalidade na qual as MPE's estão inseridas, a influência direta dos aspectos ambientais, como economia, regulação, tecnologia e sociocultural, deve ser considerada para a promoção da longevidade das MPE's. Logo o entendimento e reuso dos dados de pesquisa se tornam estratégicos, pois: a) criam uma oportunidade de representação semântica do conjunto de dados encontrados nessas pesquisas; b) possibilidade de geração de informações relevantes para um melhor planejamento da instituição; c) um entendimento mais profundo sobre o público-alvo do Sebrae em Minas Gerais; d) redução de custos, que poderiam ser revertidos em ações em prol das MPE's.

Além disso uma redução significativa da complexidade de integrar diferentes bases de dados, oferecendo um processo de extração e homogeneização de dados, permitindo encontrar e criar vinculações entre os dados internos e aqueles que possuem relação com eles.

No atual cenário de alta rivalidade no qual as MPE's do Brasil e de Minas Gerais estão inseridas, obter uma vantagem competitiva se torna vital para a longevidade das MPE's. Em uma sociedade cada vez mais baseada em data driven, a informação passa a ser um diferencial competitivo. No caso das MPE's, a informação uma vez coletada, tratada e analisada, é capaz de gerar um planejamento melhor, proposição de ações mais efetivas e direcionadas aos anseios das MPEs, e redução de custo de operação.

No contexto do Sebrae de Minas Gerais entre os anos de 2014 e 2018, foram realizadas diversas pesquisas com os empresários de micro e pequenas empresas. Entre elas constam pesquisas de origem quantitativa e com questionário estruturado. Após obtidos e analisados os resultados que a originaram a pesquisa, esses dados não são mais utilizados, por não serem integrados em uma mesma base de dados. Por isso não é possível responder a perguntas, como: "quantas vezes um mesmo cliente foi entrevistado nesse período?", ou "como os dados podem ser reorganizados de forma a responder uma nova pergunta sem a necessidade de realizar uma nova pesquisa?". Diante do exposto surge a seguinte pergunta: Como realizar a padronização e integração dos dados de pesquisa quantitativa com questionário estruturado sobre MPE's de Minas Gerais em um repositório baseado em Linked Data?

Entretanto é necessário identificar como a literatura trata os temas pequenas empresas, ontologia e integração de dados, mas vinculados uns aos outros, e não isolados. Diante disso, a pergunta principal do trabalho é: Como tem sido a evolução da bibliografia que sustenta a construção de um modelo padronização e integração dos dados de pesquisa quantitativa com questionário estruturado sobre MPE's de Minas Gerais em um repositório baseado em Linked Data? 


\section{Referencial Teórico}

\subsection{Micro e Pequenas Empresas}

Um negócio de pequeno porte é caracterizado assim por possuir apenas um ou um pequeno grupo de investidores, que opere em uma área geograficamente restrita, é pequeno comparado às empresas maiores no setor, e tem menos de 100 empregados (LONGENECKER; MOORE; PETTY, 1997, p. 43, tradução nossa).

As pequenas empresas podem ser caracterizadas por serem empresas que possuem um processo de tomada de decisão centralizado, com pouca burocracia ou controle interno, e processos de planejamento restritos (PREMKUMAR, 2003). Um exemplo é encontrado em Salazar, Soto e Mosqueda (2012, pg. 93), quando eles afirmam que "uma das principais características das pequenas empresas é que elas não têm informações financeiras úteis para tomar decisões". Isso acontece porque essas empresas normalmente usam esses dados apenas para pagar impostos e contas, devido não terem pessoal qualificado para transformar esses dados em conhecimento, para suportar o processo decisório. Essa visão é corroborada por Santos, Dorow e Beuren (2016, pg. 157), ao observarem que "na literatura as micro e pequenas empresas têm como característica a quase total ausência de instrumentos gerenciais, baseando a tomada de decisão na experiência, intuição e improvisação dos gestores".

Apesar das MPE's não terem a mesma estrutura de produção e gestão de uma empresa de médio e grande porte, que busca reduzir o custo unitário e obter ganhos de escala, a pequena empresa pode ser entendida como "a mola propulsora do desenvolvimento nacional, independentemente de seu tamanho" (FERRONATO, 2009, pg. 116). Para Longenecker, Moore e Petty (2004, p. 26), "as empresas pequenas operam em todos os setores, mas diferem enormemente em sua natureza e importância de um setor para o outro". Essa argumentação é validada pelo fato das MPE's empregarem $54 \%$ da força de trabalho do país. E essa mão de obra é local, especialmente em bairros e municípios de pequeno porte.

\subsection{Representação do Conhecimento}

Dada as características peculiares das MPE's, principalmente de gestão, a gestão do conhecimento é feita de forma intuitiva. Não há processos formalizados de registro e recuperação da informação. E um dos objetivos da organização do conhecimento é a "recuperação da informação a partir da determinação e disponibilização de pontos de acesso, apoiadas pelos recursos tecnológicos" (CATARINO; CERVANTES; ANDRADE, 2015, p. 106). Entretanto, órgãos ligados ao apoio das MPE's possuem tais recursos, tanto tecnológicos como de pessoas.

Uma vez tendo esses dois recursos é possível estruturar os processos de organização do conhecimento e da informação. Segundo Lopes e Lima (2009, p. 169), "os processos de organização do conhecimento e da informação geram duas modalidades de representação: a representação do conhecimento e a representação da informação". Nessa perspectiva a "representação do conhecimento é um processo mental (campo das idéias) responsável pela organização do conhecimento" (SALES; CAFÉ, 2009, p. 101). De acordo com Brascher e Café (2008, p. 5), a "representação da informação, é entendida como um conjunto de elementos descritivos que representam os atributos de um objeto informacional específico".

Nesse contexto, para Catarino, Cervantese e Andrade (2015, p. 106), "faz-se necessário refletir a respeito de modelos metodológicos de organização da informação e do 
conhecimento que atendam às necessidades informacionais de usuários no âmbito de um sistema de informação especializado". Esses modelos de representação do conhecimento, podem ser tesauros e ontologias, "que representam uma parte do mundo das idéias (representação do conhecimento), e são aplicados nas atividades de representação da informação" (SALES; CAFÉ, 2009, p. 101). Logo, a "representação do conhecimento reflete um modelo de abstração do mundo real, construído para determinada finalidade" (BRASCHER; CAFÉ, 2008, p. 6).

\subsection{Ontologia e Web Semântica}

Nesse sentido, de acordo com Almeida (2014, pg. 243), "dentre as diversas técnicas disponíveis para organização da informação e do conhecimento, a aplicação de ontologias tem recebido cada vez mais atenção". Uma ontologia define um vocabulário comum para pesquisadores que precisam compartilhar informações em um domínio, e isto inclui definições interpretáveis por máquina, dado os conceitos básicos de um domínio e as relações entre eles (NOY; MCGUINNESS, 2001, p. 1, tradução nossa).

Entretanto, como aponta Mendonça e Soares (2017, pg.45), o "processo de construção de uma ontologia é complexo, pois envolve a criação de modelos semânticos ou descrições simplificadas da realidade de um dado domínio". E a semântica lida com o significado de sinais e símbolos ou, em outras palavras, com as informações por trás de um dado (GUIZZARDI, 2005, p. 19, tradução nossa).

Esse debate sobre ontologia e semântica leva a outra discussão muito importante na atualidade, a Web Semântica. O termo foi cunhado por Tim Berners-Lee em 1994. Desde o princípio da World Wide Web (a Web) o objetivo foi vincular o máximo de coisas por meio de links (GANDON, 2018, tradução nossa), porém com a evolução da WEB, houve a necessidade de melhorias, com informações semânticas mais orientadas à máquina, permitindo um processamento mais sofisticado (BERNERS-LEE et al., 1994, tradução nossa). Logo, "a Web Semântica estende a web clássica, provendo uma estrutura semântica para páginas web, a qual permite tanto agentes humanos quanto agentes de software possam entender o conteúdo presente em páginas web" (ISOTANI; BITTENCOURT, 2015, p. 27). De acordo Nowack (2009), a Web Semântica é composta por uma pilha de tecnologias, como Dados Conectados, Conceitos \& Abstrações, e Especificações e Soluções, que mostram "toda a complexidade e as tecnologias envolvidas com a área" (ISOTANI; BITTENCOURT, 2015, p. 29). Essa pilha de tecnologias da Web Semântica é demonstrada na Figura 01.

Diante disso, ontologia pode ter uma representação formal, voltada para ser consumida por máquinas, e uma representação gráfica, voltada para compreensão humana (ISOTANI; BITTENCOURT, 2015). Ao longo do tempo, esse contexto foi potencializado pela padronização das linguagens de ontologia de Web Semântica, criando mais dados e informações. Isso pode ser denominado "problema de Big Data", de Cox e Ellsworth (1997), que é um desafio "para sistemas de computadores, onde os conjuntos de dados são geralmente muito grandes, sobrecarregando a memória principal, disco local e até disco remoto" (COX; ELLSWORTH, 1997, p. 2, tradução nossa). 
Figura 01 - Pilha de Tecnologias da Web Semântica

The Semantic Web Technology Stack

(not a piece of cake...)

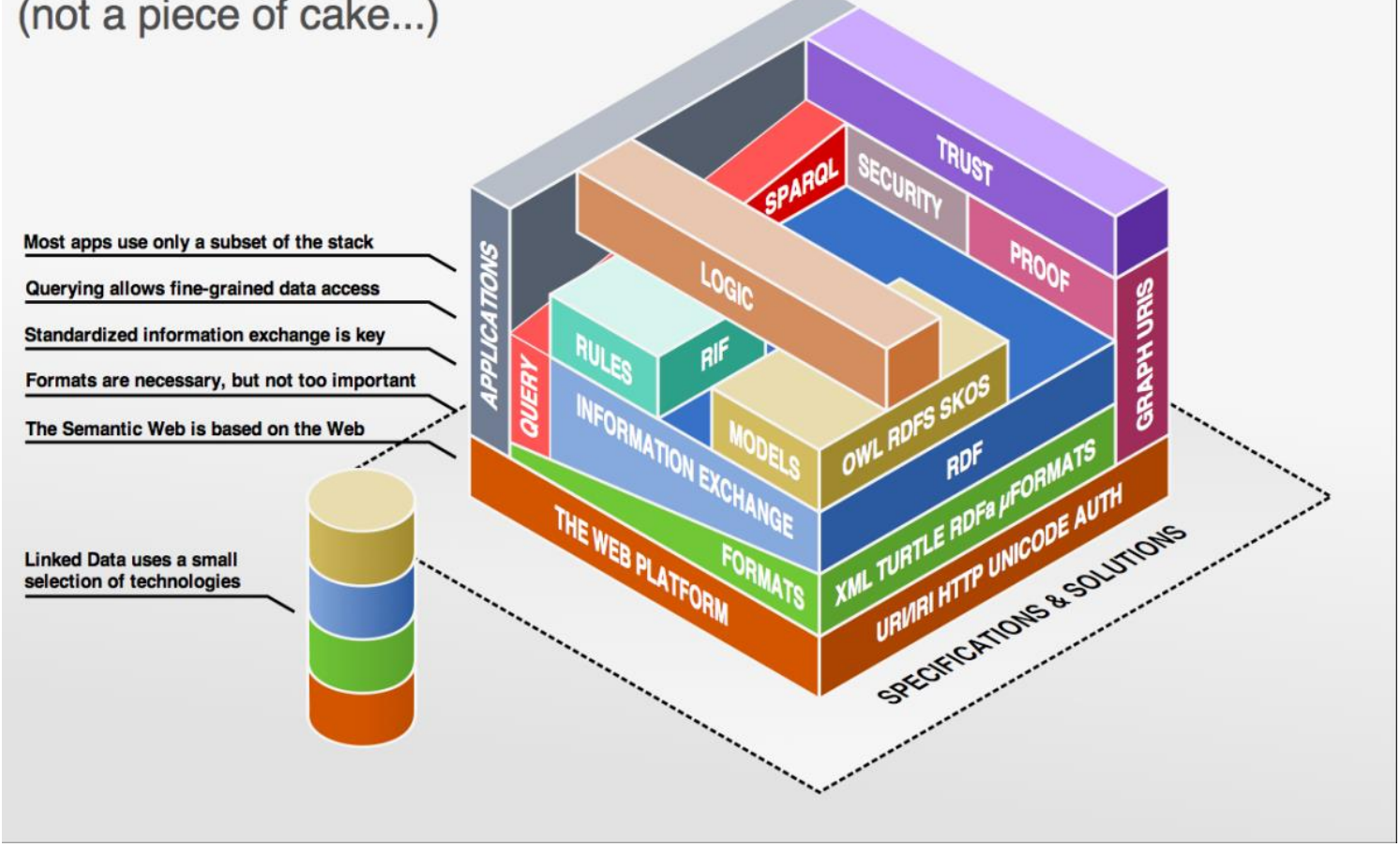

Fonte: Nowack, 2009

Para Jain et al (2016, p.1), Big Data "refere-se especificamente a um conjunto de dados tão grandes ou complexos que os aplicativos tradicionais de processamento de dados não são suficientes". A Pilha de Tecnologias da Web Semântica de Nowack (2009), na qual ontologia está presente, como modelo conceitual, linguagem de programação e softwares, traz de forma subjacente o conceito Big Data, aplicado a um domínio específico do conhecimento. Porém isso só é possível uma vez que os dados estão conectados entre si. E a ontologia é o arcabouço técnico e teórico que dá base á essa integração de dados.

\subsection{Integração de Dados e Linked Data}

De acordo com Farinelli e Elkin (2017, pg. 125), "atualmente, o maior desafio das organizações é a necessidade de integração de informações com seus parceiros internos e externos”. Para isso é necessário um modelo de integração de dados, que necessita de um modelo conceitual. Esse modelo conceitual facilita e orienta como os dados serão organizados e recuperados.

Segundo Castro e Jesus (2018, p. 150), a proposta de Linked Data é "a ligação de dados representados em um formato padrão, a partir da utilização de links semânticos, da utilização de ferramentas e de práticas propostas pelo movimento". Toda a tendência de Linked Data é baseada em princípios e metodologias para tecer uma Web de dados. Os conceitos, princípios técnicos e avanços dos Linked Data na Web foram estudados e documentados ao longo dos anos (GANDON, 2018, p. 28, tradução nossa). De acordo com Berners-Lee (2006), os princípios do Linked Data são compostos por quatro recomendações básicas, sendo: 1 - Uso 
de Uniform Resource Identifier (URIs); 2. Uso de HTTP URIs; 3. Uso do Resource Description Framework (RDF); e 4. Inclusão de links para outros URIs.

Para Isotani e Bittencourt (2015, p. 23), neste contexto, "surge a Web de dados, na qual os recursos estão conectados por URIs, e informações adicionais são disponibilizadas para permitir que as máquinas possam compreender melhor os dados contidos nestes recursos". Esse contexto de Linked Data garante a recuperação dos conteúdos e ainda, a interoperabilidade entre as diversas fontes de informação em um determinado ambiente (CASTRO; JESUS, 2018, p. 149).

Por fim, a importância de utilizar Integração de Dados e Linked Data se justifica "pela necessidade de estruturação dos dados em formato aberto, legível por máquina e com ligações semânticas que possam enriquecer seus conteúdos, fornecendo contexto ao usuário" (BRANDT; VIDOTTI; SEGUNDO, 2018, p. 150).

\section{$3 \quad$ Metodologia}

A metodologia utilizada para captar o que a bibliografia trata do tema desse trabalho foi a Systematic Review Literature (SLR) ou Revisão Sistemática da Literatura (RSL). A RSL é composta por três etapas: (1) planejamento da revisão, (2) condução da revisão e (3) relatório da revisão (KITCHENHAM, 2004; KITCHENHAM et al., 2010).

Os termos utilizados foram: "small business", "ontology", "data integration", "linked data" e "semantic dictionary". Esses termos foram utilizados para captar a maior quantidade de ocorrências possíveis, pois a produção acadêmica nacional indexa os materiais também na língua inglesa. As bases de dados pesquisadas com suas respectivas URL's (acessadas por meio do Portal Capes) são apresentadas no quadro a seguir:

\section{Quadro 01 - Bases de dados pesquisadas}

\begin{tabular}{|l|l|}
\hline \multicolumn{1}{|c|}{ Base de dados } & \multicolumn{1}{c|}{ URL } \\
\hline Web of Science & $\begin{array}{l}\text { http://apps- } \\
\text { webofknowledge.ez27.periodicos.capes.gov.br/WOS_GeneralSearch_i } \\
\text { nput.do?product=WOS\&SID=8B4g8QVWtTVIReHsakx\&search_mo } \\
\text { de=GeneralSearch }\end{array}$ \\
\hline Google Scholar & https://scholar-google-com-br.ez27.periodicos.capes.gov.br/ \\
\hline \multicolumn{1}{|c|}{ Fonte: Elaborado pelo autor }
\end{tabular}

Foram utilizadas três combinações de strings, sendo: a) "small business" and ontology and "data integration"; b) "small business" and ontology and "linked data"; c) "small business" and ontology and "semantic dictionary".

\section{$4 \quad$ Analise dos dados}

A partir do resultado dessa pesquisa no portal Google Scholar foi possível constatar que há bastante produção acadêmica entre 2000 e 2019, com esses termos. Mas ao pesquisar relacionando os termos entre si evidenciou que há pouca produção acadêmica. A pesquisa se concentrou no portal Google Scholar, por retornar uma quantidade maior de ocorrências com os termos cruzados. O resultado dessa pesquisa está demonstrado no Gráfico 01. 


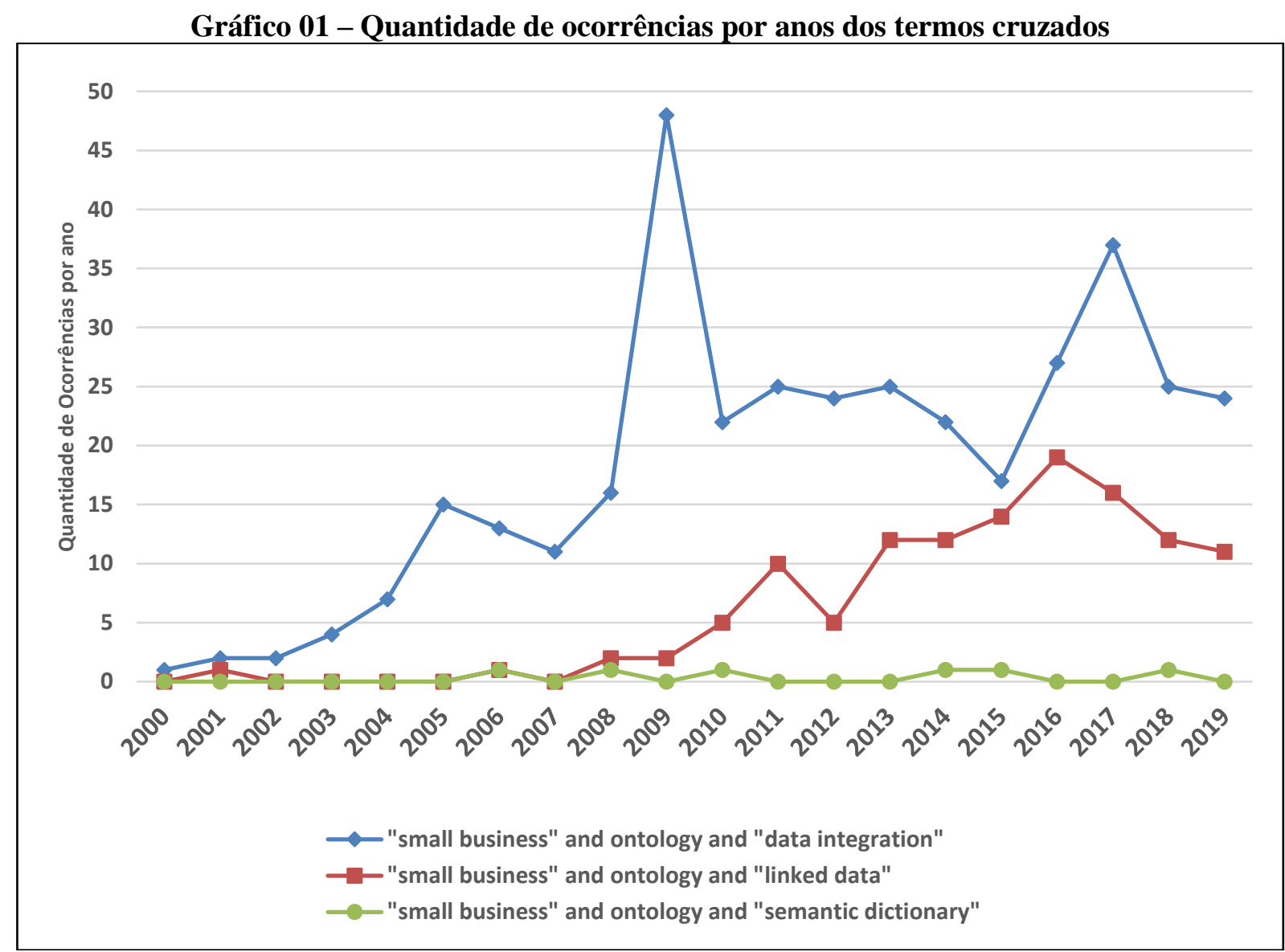

Fonte: Elaborado pelo autor

Os dados mostram um aumento na produção cientifica voltada para as MPE's, relacionada à ontologia e integração de dados. Os dados também mostram uma evolução na quantidade de pesquisas que relacionam MPE's, ontologia e linked data. Apesar do total absoluto ser considerado baixo, há uma evolução, o que evidência uma tendência dessas temáticas serem mais pesquisadas nos próximos anos.

Para identificar trabalhos correlatos, foi pesquisado no site da CAPES (https://www.periodicos.capes.gov.br) termos relacionados ao projeto proposto. A pesquisa concentrou em artigos, publicados entre os anos 2000 e 2019, nos seguintes tópicos: Small \& Medium Sized Enterprises-Sme; Small Business; Knowledge Management; Interoperability; Semantics; Engineering; Entrepreneurship; Economics; Ontology; Business; Small Business. Esses tópicos foram selecionados para restringir a pesquisa em temáticas de interesse do projeto. Ao realizar a com os termos "small business" and "ontology", e "small business" and "data integration", foram retornados 35 artigos. No quadro 02 estão listados os principais artigos com maior correlação ao propósito desse projeto.

Quadro 02 - Relação de trabalhos correlatos

\begin{tabular}{|l|l|c|}
\hline Artigo & Autor & Ano \\
\hline Ontology mapping using description logic and & Kumar, Sri ; Harding, Ja & 2013 \\
\hline
\end{tabular}




\begin{tabular}{|c|c|c|}
\hline bridging axioms & & \\
\hline $\begin{array}{l}\text { Semantic search for matching user requests } \\
\text { with profiled enterprises }\end{array}$ & $\begin{array}{l}\text { Formica, Anna ; Missikoff, } \\
\text { Michele; Pourabbas, Elaheh ; } \\
\text { Taglino, Francesco }\end{array}$ & 2013 \\
\hline $\begin{array}{l}\text { A Human-Centered Semantic Service Platform } \\
\text { for the Digital Ecosystems Environment }\end{array}$ & $\begin{array}{l}\text { Dong, Hai ; Khadeer Hussain, } \\
\text { Farookh ; Chang, Elizabeth } \\
\text { Dong, Hai }\end{array}$ & 2010 \\
\hline $\begin{array}{l}\text { New Approach for Optimal Semantic-Based } \\
\text { Context-Aware Cloud Service Composition for } \\
\text { ERP }\end{array}$ & Alti, Adel & 2018 \\
\hline $\begin{array}{l}\text { How to deal with knowledge management } \\
\text { misalignment: a taxonomy based on a 3D fuzzy } \\
\text { methodology }\end{array}$ & $\begin{array}{l}\text { Centobelli, Piera ; Cerchione, } \\
\text { Roberto ; Esposito, Emilio }\end{array}$ & 2018 \\
\hline $\begin{array}{l}\text { An Ontology-centered Approach for Designing } \\
\text { an Interactive Competence Management } \\
\text { System for IT Companies }\end{array}$ & $\begin{array}{l}\text { Niculescu, Cristina ; Trausan- } \\
\text { Matu, Stefan }\end{array}$ & 2009 \\
\hline $\begin{array}{l}\text { Integration of Technology Management and } \\
\text { Selected Specifics of Best Technology- } \\
\text { Integrated Companies }\end{array}$ & Jemala, Marek & 2012 \\
\hline $\begin{array}{l}\text { Semantic parameters to manage an innovation } \\
\text { network using managing as designing } \\
\text { approach: the virtual innovation society } \\
\text { network case }\end{array}$ & $\begin{array}{l}\text { Gattaz, Cristiane } \quad \text { Chaves } \\
\text { Cruvinel, Paulo Estevao } \\
\text { Piscopo, Marcos Roberto }\end{array}$ & 2014 \\
\hline
\end{tabular}

Fonte: Elaborado pelo autor

Os dados mostram que há pouca produção acadêmica que relaciona MPE's e ontologia, ligados a integração de dados, linked data e dicionário semântico. Entretanto, os dados mostram uma evolução positiva na quantidade de pesquisas que relacionam "small business" e "ontology" com "data integration" e "linked data".

Diante disso é possível deduzir que há uma carência de pesquisas voltadas para ontologia e MPE's, principalmente no Brasil. Uma vez que as MPE's são importantes para a economia e desenvolvimento do pais, estudos de ontologia e integração de dados devem ser desenvolvidos para suportar os processos produtivos e de planejamento das instituições de apoio e órgãos de fomento ligados a MPE's.

\section{Contribuições}

A expectativa de contribuições é relevante para as MPE's, pois uma vez tendo esse sistema consolidado, as empresas desfrutariam de um repositório informacional para trabalhar o ambiente empresarial. Um outro ponto é oferecer um recurso de pesquisa para universidades focadas na pesquisa de MPE's. Dessa forma os pesquisadores poderiam utilizar os dados armazenados nessa estrutura, como inserir dados de pesquisa. Isso possibilitaria o uso e o reuso de dados de pesquisa. 
Por isso o uso de ontologia, pois permite compartilhar um entendimento comum da estrutura das informações entre pessoas ou agentes de software, e ativar a reutilização do conhecimento do domínio. Também permite tornar explícitas as suposições de domínio, separar o conhecimento do domínio do conhecimento operacional, e analisar o conhecimento do domínio (NOY; MCGUINNESS, 2001, p. 1, tradução nossa).

Ao entender que ontologia é parte e base de algo maior, como a Web Semântica, a representação da informação de um domínio passa a ser mais tangível. Segundo Hitzler, Krötzsch e Rudolph (2009, p. 335, tradução nossa), não há escassez de possíveis cenários futuros de casos de uso para tecnologias da Web Semântica, desde a integração de informações sobre inteligência ambiental até sistemas especializados.

Logo, as opções de aplicação são inúmeras. Uma área de aplicação de alto potencial, por exemplo, é a Pesquisa Semântica, que se refere ao uso de metadados e conhecimentos básicos na forma de ontologias para aprimorar a pesquisa de informações na World Wide Web e nas intranets corporativas (HITZLER; KRÖTZSCH; RUDOLPH, 2009, p. 348, tradução nossa).

Uma vez tendo esse contexto aplicada para a gestão das MPE's, monitoramento e entendimento do ambiente das MPE's, a proposição de estratégias e politicas tendem a ser mais assertivas e focadas nas necessidades dessas empresas. Visa alcançar um conhecimento mais aprofundado sobre como solucionar problemas de negócios, recuperação de informações em ambientes direcionados por dados, e contribuir para o avanço de pesquisas no campo da gestão do conhecimento focada em MPE's.

\section{Referências}

ALMEIDA, Maurício Barcellos. Uma abordagem integrada sobre ontologias: Ciência da Informação, Ciência da Computação e Filosofia. Perspectivas em Ciência da Informação, v.19, n.3, p.242-258, jul./set. 2014.

BERNERS-LEE, Tim; CAILLIAU, Robert; LUOTONEN, Ari; NIELSEN, Henrik Frystyk; SECRET, Arthur. The worldwide web. Communications of the ACM, Vol. 37, No. 8, pp. 76-82, 1994. Retrieved from http://doi.acm.org/10.1145/179606.179671

BERNERS-LEE, Tim. Linked data, 2006. Disponível em: <https://www.w3.org/DesignIssues/ LinkedData.html>. Acesso em: 17 set. 2019.

BRANDT, Mariana Baptista; VIDOTTI, Silvana Aparecida Borsetti Gregorio; SEGUNDO, José Eduardo Santarem. Modelo de dados abertos conectados para informação legislativa. Inf. \& Soc.:Est., João Pessoa, v.28, n.2, p. 149-161, maio/ago. 2018.

BRASCHER, Marisa; CAFÉ, Lígia. Organização da informação ou organização do conhecimento? In: ENCONTRO NACIONAL DE PESQUISA EM CIÊNCIA DA INFORMAÇÃO, 9., 2008, São Paulo. Anais eletrônicos... São Paulo: ANCIB, USP, 2008. 
CASTRO, Fabiano Ferreira de; JESUS, Ananda Fernanda de. Adequando dados bibliográficos ao Linked Data. Inf. \& Soc.:Est., João Pessoa, v.28, n.3, p. ?-?, set./dez. 2018

CATARINO Maria Elisabete; CERVANTES, Brígida Maria Nogueira; ANDRADE Ilza Almeida de. A representação temática no contexto da WEB semântica. Inf. \& Soc.:Est., João Pessoa, v.25, n.3, p. 105-116, set./dez. 2015.

COX, Michael; ELLSWORTH, David. Application Controled Demand Paging for Out-orCore Visualization. NASA, Ames Research Center, 1997.

DAVENPORT, Thomas H.; PRUSAK, Lawrence. Conhecimento empresarial: como as organizações gerenciam o seu capital intelectual. Rio de Janeiro: Campus, 2003.

FARINELLI, Fernanda; ELKIN, Peter L. Construção de ontologia na prática: um estudo de caso aplicado ao domínio obstétrico. Ci.Inf., Brasília, DF, v.46 n.1, p.123-141, jan./abr. 2017.

GANDON, Fabien. A Survey of the First 20 Years of Research on Semantic Web and Linked Data. Revue des Sciences et Technologies de 1'Information - Série ISI : Ingénierie des Systèmes d'Information, Lavoisier, 2018.

GUIZZARDI, Giancarlo. Ontological foundations for structural conceptual models. Enschede: Telematica Instituut / CTIT, 2005.

HITZLER, Pascal; KRÖTZSCH, Markus; RUDOLPH, Sebastian. Foundations of Semantic Web Technologies. [Online] 2009. Disponível em: <http://people.mpiinf.mpg.de/ dstepano/KRSW/literature/SWTechnologies.pdf>

ISOTANI,Seiji; BITTENCOURT, Ig Ibert. Dados abertos conectados. São Paulo: Novatec Editora, 2015.

JAIN, Priyank; GYANCHANDONI, Manasi; KHARE, Nilay. Big Data privacy: a technological perspective and review. Journal of Big Data, 2016.

KITCHENHAM, B. Procedures for Performing Systematic Reviews. Joint Technical Report, Computer Science Department, Keele University (TR/SE-0401) and National ICT Australia Ltd. NICTA Technical Report 0400011T.1, 2004.

KITCHENHAM, B. et al. Systematic literature reviews in software engineering - A tertiary study. Information and Software Technology, Elsevier B.V., v. 52, n. 8, p. 792-805, ago. 2010 . 
LOPES, Patrícia; LIMA, Gercina Angela. Estratégias de Organização, Representação e Gestão de Trilhas de Aprendizagem: uma revisão sistemática de literatura. Perspect. ciênc. inf., Belo Horizonte, v. 24, n. 2, p. 165-195, June 2019.

LONGENECKER, Justin G.; MOORE, Carlos W.; PETTY, William J. Administração de pequenas empresas. São Paulo, Makron Books, 2004.

MENDONÇA, Fabrício Martins; SOARES, António Lucas. Construindo ontologias com a metodologia ontoforinfoscience: uma abordagem detalhada das atividades do desenvolvimento ontológico. Ci.Inf., Brasília, DF, v.46 n.1, p.43-59, jan./abr. 2017.

NOY, Natalya; MCGUINNESS, Deborah. Ontology Development 101: A Guide to Creating Your First Ontology. Knowledge Systems Laboratory, 32, 2001.

NOWACK, Bernd. The Semantic Web Technology Stack (not a piece of cake...). Linked Data Developer. [Online] $2009 . \quad$ Disponível em: <http://linkeddatadeveloper.com/Projects/Linked-Data/media/fig11.2.png>.

PREMKUMAR, G. A meta-analysis of research on information technology implementation in small business. Journal of Organizational Computing and Electronic Commerce, USA, v.13, n.2, p.91-121, June 2003.

SALAZAR, Alejandra López; SOTO, Ricardo Contreras; MOSQUEDA, Rafael Espinosa. The Impact of Financial Decisions and Strategy on Small Business Competitiveness. Global Journal of Business Research, Volume 6, Number 2 - 2012.

SALES, Rodrigo de; CAFE, Lígia. Diferenças entre tesauros e ontologias. Perspect. ciênc. inf., Belo Horizonte, v. 14, n. 1, p. 99-116, Apr. 2009.

SANTOS, Vanderlei dos Diego; DOROW, Roberto; BEUREN, Ilse Maria. Práticas gerenciais de micro e pequenas empresas. Revista Ambiente Contábil - ISSN 2176-9036 - UFRN Natal-RN. v. 8. n. 1, p. 153 - 186, jan./jun. 2016.

SEBRAE. O Público do Sebrae. Brasília, DF. Junho/2018.

SEBRAE. Observatório Internacional Sebrae, 2019. Disponível em: http://ois.sebrae.com.br/wp-content/uploads/2012/11/Estados-Unidos1.pdf.

SEBRAE. 2019. em: 〈http://www.sebrae.com.br/sites/PortalSebrae/canais_adicionais/conheca_quemsomos $>$

SILVA, Ricardo V.; NAVES, Ana. Gestão de empresas na era do conhecimento. São Paulo: Serinews, 2004. 
TEIXEIRA, Livia Marangon Duffles; ALMEIDA, Maurício Barcellos. Princípios ontológicos no suporte a terminologias clínicas: método e ontologia para reorganização da Classificação Internacional de Doenças. Ci.Inf., Brasília, DF, v.48 n.1, p.94-112, jan./abr. 2019. 